Clinical Study

\title{
Comparison of Glycomacropeptide with Phenylalanine Free-Synthetic Amino Acids in Test Meals to PKU Patients: No Significant Differences in Biomarkers, Including Plasma Phe Levels
}

\author{
Kirsten K. Ahring $\mathbb{D}^{1,2,3}$ Allan M. Lund, ${ }^{2,3}$ Erik Jensen, ${ }^{4}$ Thomas G. Jensen, ${ }^{5}$ \\ Karen Brøndum-Nielsen, ${ }^{1}$ Michael Pedersen, ${ }^{6}$ Allan Bardow, ${ }^{7}$ \\ Jens Juul Holst $\mathbb{D}^{8},{ }^{8}$ Jens F. Rehfeld, ${ }^{9}$ and Lisbeth B. Møller ${ }^{2}$ \\ ${ }^{1}$ The PKU Clinic, Kennedy Centre, Centre for Paediatric and Adolescent Medicine, Copenhagen University Hospital, Rigshospitalet, \\ Copenhagen, Denmark \\ ${ }^{2}$ Department of Clinical Genetics, Applied Human Molecular Genetics, Kennedy Center, Rigshospitalet, Denmark \\ ${ }^{3}$ Centre for Inherited Metabolic Diseases, Centre for Paediatric and Adolescent Medicine, Copenhagen University Hospital, \\ Rigshospitalet, Copenhagen, Denmark \\ ${ }^{4}$ Arla Foods Ingredients Group P/S, Viby J, Denmark \\ ${ }^{5}$ Department of Biomedicine, Aarhus University, Aarhus, Denmark \\ ${ }^{6}$ Department of Clinical Medicine, Aarhus University Hospital, Aarhus, Denmark \\ ${ }^{7}$ Department of Odontology, Copenhagen University, Copenhagen, Denmark \\ ${ }^{8}$ Institute of Clinical Medicine, Copenhagen University Hospital, Rigshospitalet, Copenhagen, Denmark \\ ${ }^{9}$ Department of Clinical Biochemistry, University of Copenhagen, Rigshospitalet, Copenhagen, Denmark
}

Correspondence should be addressed to Kirsten K. Ahring; kka@kennedy.dk

Received 14 July 2017; Accepted 11 October 2017; Published 8 January 2018

Academic Editor: Ina Knerr

Copyright ( 2018 Kirsten K. Ahring et al. This is an open access article distributed under the Creative Commons Attribution License, which permits unrestricted use, distribution, and reproduction in any medium, provided the original work is properly cited.

\begin{abstract}
Introduction. Management of phenylketonuria (PKU) is achieved through low-phenylalanine (Phe) diet, supplemented with lowprotein food and mixture of free-synthetic (FS) amino acid (AA). Casein glycomacropeptide (CGMP) is a natural peptide released in whey during cheese-making and does not contain Phe. Lacprodan ${ }^{\circledR}$ CGMP-20 used in this study contained a small amount of Phe due to minor presence of other proteins/peptides. Objective. The purpose of this study was to compare absorption of CGMP-20 to FSAA with the aim of evaluating short-term effects on plasma AAs as well as biomarkers related to food intake. Methods. This study included 8 patients, who had four visits and tested four drink mixtures (DM1-4), consisting of CGMP, FSAA, or a combination. Plasma blood samples were collected at baseline, 15, 30, 60, 120, and 240 minutes (min) after the meal. AA profiles and ghrelin were determined 6 times, while surrogate biomarkers were determined at baseline and 240 min. A visual analogue scale (VAS) was used for evaluation of taste and satiety. Results. The surrogate biomarker concentrations and VAS scores for satiety and taste were nonsignificant between the four DMs, and there were only few significant results for AA profiles (not Phe). Conclusion. CGMP and FSAA had the overall same nonsignificant short-term effect on biomarkers, including Phe. This combination of FSAA and CGMP is a suitable supplement for PKU patients.
\end{abstract}

\section{Introduction}

Phenylketonuria (PKU) is an inborn error of metabolism. If left untreated, severe brain damage will occur [1-3]. The primary aim of treatment of those suffering from PKU is to control the blood phenylalanine (Phe) concentration in order to prevent neurological damage [2]. PKU treatment is based on a low-protein (LP) diet in combination with freesynthetic (FS) amino acid (AA) supplements without Phe and enriched with vitamins, minerals, trace elements, and in 
some products also fat and carbohydrates [1, 4, 5]. Dietary AA supplements are administered to obtain optimal metabolic control by ensuring adequate levels of essential AAs. Diet for life is recommended [6]. Compliance becomes a challenge over time, especially in adolescence, and they are often related to disagreeable taste and the current limitations in available dietary products $[7,8]$. In order to achieve better compliance, the formulation of the AA supplement should satisfy the need for better taste and easier management [9]. Therefore, alternatives to conventional treatment are investigated.

Casein glycomacropeptide (CGMP) is a 64-amino acid peptide from cheese whey, which is rich in specific essential AAs and is the only known natural protein free from Phe [10-14]. Hence, CGMP is an alternative source of protein for PKU patients. In this study, we tested the product Lacprodan CGMP-20, a highly purified CGMP product with minimum 95\% CGMP and a low level of Phe $(0.16 \mathrm{~g} / 100 \mathrm{~g}$ AA) and above $78 \%$ protein. The residual amount of Phe is due to the presence of minor amounts of other proteins/ peptides. However, to ensure the supplement is nutritionally adequate, it requires supplementation of the following AAs to meet the standards of similar PKU supplements: tyrosine (Tyr), tryptophan (Trp), arginine (Arg), histidine (His), leucine (Leu), lysine (Lys), and methionine (Met) [15-17]. Lacprodan CGMP-20 will be referred to as CGMP in this paper.

In this single-blinded, prospective, crossover intervention study, we investigated the utilization and metabolic short-term effect on absorption of pure CGMP-20, FSAA, and a combination of both, all consumed with a standardized meal, by comparing selected relevant surrogate biomarkers. We also evaluated taste and satiety. A second aim was to investigate whether the small amount of Phe in CGMP-20 affected the plasma Phe concentration significantly.

\section{Materials and Methods}

2.1. Protocol. The study was approved by the National Committee on Health Research Ethics prior to the study (ID H-3-2014-115), and all participants gave written consent prior to start of the study.

2.2. Patient Recruitment. Patients were contacted by mail and invited to participate. They were recruited from the clinical PKU database, including all PKU patients diagnosed and living in Denmark. Patients received compensation for lost wages and travel expenses. Participants in the project were invited to participate if they met the following inclusion criteria: diagnosis of classical PKU confirmed by mutation analysis and a known phe tolerance (12-20 mg/kg) [18-20], age $\geq 15$ years at inclusion, had received treatment with a protein-restricted diet since the neonatal period, and were willing and able to visit the PKU clinic four times. The period between visits varied from 24 hours to 1 month, since we estimated this to be sufficient time for a wash-out period [21, 22 ]. Exclusion criteria were $(1)<15$ years at inclusion, (2) had not followed the dietary treatment continuously, (3) had a second chronic disease or condition, which potentially could influence the PKU treatment and outcome, (4) treated with $\mathrm{BH} 4$, or (5) pregnant, nursing, or planning to become pregnant. Eight patients accepted to participate.

2.3. Study Design. The primary purpose of this study was to compare the absorption rate and absorbed amount of peptide-bound AAs (CGMP-20*) (both in its pure form or supplemented with selected FSAAs) with an almost identical mixture of FSAAs with the aim of evaluating the short-term effect on Phe and other AAs as well as biomarkers related to food intake. All patients had four visits in the PKU clinic. The patients consumed a different drink mixture (DM1, DM2, DM3, and DM4) in random order at each visit (blinded to the participants). The order of the DM was randomized by a doctor at the PKU clinic, who was not otherwise involved in the study. CGMP/AA supplements: four different AA sources were tested. DM1: Lacprodan CGMP-20. DM2: FSAA (equivalent AA profile as DM1). DM3: Lacprodan CGMP-20 and synthetic AA. DM4: FSAA (equivalent AA profile as DM3 but without Phe). DM2 had the same AA profile as DM1 consisting of pure CGMP in order to evaluate this; DM3 consisted of CGMP supplemented with FSAA to make it nutritionally adequate and suitable for patients with PKU and had a similar AA profile as DM4 (though this was without the $0.16 \mathrm{~g}$ Phe/ $100 \mathrm{~g} \mathrm{AA}$ present in CGMP). The crossover study design made it possible to evaluate the effect of the Phe content in GMP. Patients arrived fasting to the clinic in the morning and then subjected to the first venous blood samples $(4 \mathrm{ml})$ at time 0 . Subsequently, blood samples were drawn at 15, 30, 60, 120, and 240 minutes ( $\mathrm{min}$ ) after finishing the meal. Taste was evaluated right after consumption. At the end of the visit, all participants were asked to evaluate satiety.

The test meals consisted of a few selected food items (homemade LP bread, butter, and jam) with individually calculated amounts of fat and carbohydrates and only a minimal content of protein and Phe from LP bread. The test meal was consumed in combination with DM, and the individual intake was designed to cover $25 \%$ of the daily requirement. In each meal, the total content of protein was equivalent to $25 \%$ of $1 \mathrm{~g}$ per $\mathrm{kg}$ body weight per day $(1 \mathrm{~g} / \mathrm{kg} / \mathrm{d})$. The composition of the meal was calculated after the Nordic Nutrition Recommendations (NNA) and met the criteria for sex, age, and weight: $10-15 \%$ from protein, 30-35\% from fat, and 50-60\% from carbohydrate. After completing the trial, all participants would continue their usual diet and AA supplements. An example of a meal is shown in Table 1, and the content of DM1-4 is given in Table 2.

2.4. Blood Samples. All participants had the following blood samples drawn at start (time 0) before consuming the meal/DM and at the end of the study (240 min): glucose, insulin, glucagon-like peptide-1(GLP-1), blood urea nitrogen (BUN), peptide tyrosine-tyrosine (PYY), cholecystokinin (CCK), ghrelin, and AA profiles. Furthermore, ghrelin and AA profiles were (besides at time 0) also measured at 15, 30, 60, 120, and $240 \mathrm{~min}$. 
TABLE 1: Example of a test breakfast meal including DM (calculated individually for each participant).

DM (CGMP-20, AA, or CGMP+AA)

2 slices $(80 \mathrm{~g})$ of LP bread

Butter (20 g)

Jam $(20 \mathrm{~g})$

2.4.1. BUN, Glucose, Insulin, Ghrelin, GLP-1, PYY, and CCK. 2-4 $\mathrm{ml}$ of blood was collected for each biomarker and handled immediately according to protocols [23-28], placed on ice, spinned at $2500 \mathrm{~g}$ for $10 \mathrm{~min}$ at $4^{\circ} \mathrm{C}$, and transferred to specific glasses for further analyses. EDTA glasses for (1) GLP-1 were added $200 \mu \mathrm{l}$ DPP-IV inhibitor and for (2) ghrelin were added $100 \mu \mathrm{l}$ Pefabloc by using a $0.5 \mathrm{ml}$ syringe The samples for the total plasma AA profile were frozen at $-80^{\circ} \mathrm{C}$ and sent on dry ice for quantitative analysis of AA using the stable-isotope dilution technique and HPLC-MS/MS [29].

2.5. Visual Analogue Scale (VAS). This scale (http://www. vastranslator.com) was presented to patients as a horizontal line, ranking from "very hungry" ("0") to "very satisfied" ("100") and from "bad taste" ("0") to "good taste" ("100") as an application (APP) on iPad. The patients were asked to evaluate the taste of the DM shortly after intake and after $240 \mathrm{~min}$ to determine the level of satiety.

2.6. Body Mass Index (BMI) and Fat Percentage. These were measured at visit 1 with a body fat monitor (BF306, Omron Healthcare, USA).

2.7. Diet Registration. The patients had filled out a dietary record covering the 24 hours before each visit. They were instructed to eat similar food items every time to ensure that the Phe level would be within the same range the day after. The registrations were calculated with Dankost (http:// dankost.dk/english).

2.8. Statistical Methods. All calculations were performed using the software SPSS 22 or Microsoft Excel 2010 for windows. Paired and unpaired $t$-tests and one- and two-way ANOVA performed at a significance level of $\alpha=0.05$ were used. The statistical significance level was at $p<0.05$.

\section{Results}

3.1. Clinical Protocol. Eight patients (seven females, one male), age 15-48 years (mean $33.25 \pm$ SD 11.21), weight $47-85 \mathrm{~kg}$ (mean $72.8+\mathrm{SD} 15.9$ ), and height $162-179 \mathrm{~cm}$ (mean 170.1 + SD 5.4) were included in the study. Data for each patient are presented in Table 3. Six out of eight (75\%) patients completed the study. Patient ID\#4 had difficulties eating the meal within $15 \mathrm{~min}$ at visit one, which subsequently delayed blood sampling up to $10 \mathrm{~min}$ for each blood sample. Patient ID\#4 completed day 1 and day 2 as planned, but on the third day, blood sampling was impossible, and for that reason, visit 4 was cancelled and the patient excluded. Patient
ID\#5 completed visit 1 and visit 2 but was unable to complete day 3 and day 4 due to health problems.

3.2. Diet Registration. The 24-hour diet record confirmed similar intake for each patient prior to the four test days.

3.3. Test Meals and DM. Intake of protein from DM supplement was $25 \%$ of the daily recommendation of $1 \mathrm{~g}$ protein/kg/day (mean volume $151.8 \mathrm{~g}$ (range 97.9-195.8)), and the test meal provided fat and carbohydrates (Table 4). All patients complied with the intake.

3.4. AA Profiles. AA profiles were compared in subgroups (DM1 versus DM2 and DM3 versus DM4), since the respective groups were identical concerning the AA composition. Statistical results were calculated between these subgroups.

The area under the curve (AUC) (adjusted for baseline) for total AA demonstrated insignificant differences between DM1 and DM2 $(p=0.852)$ as well as between DM3 and DM4 $(p=0.06)$.

We did find significant differences for the following individual AA for AUC: DM1 and DM2: Lys $(p=0.0287)$, Asn $(p=0.0210)$, and Asp $(p=0.0047)$ and DM3 and DM4: citrulline $(p=0.0162)$. Results for all the AUC for each AA are presented in Table 5. The highest value for the AUC for the individual AA was Ala, Val, Ile, and Asp (DM1), Pro (DM2), and Leu (DM3).

3.5. Plasma Concentrations of AA. The peak plasma concentrations for AAs (given as mean values in percentage of the premeal level) were as follows: DM1: the peak serum concentrations were reached after $30 \mathrm{~min}$ for 19 of $21(90 \%)$ AAs. Glu reached a peak after $15 \mathrm{~min}$; Tyr only decreased compared to baseline. DM2: four AAs peaked after $30 \mathrm{~min}$, while 15 (71\%) AAs peaked after $15 \mathrm{~min}$. Citrulline peaked after $60 \mathrm{~min}$; Tyr decreased compared to time 0 . DM3: majority of the AAs peaked after 30 min (67\%) except for Asp, Met, and Gln, where the peaks were reached after $15 \mathrm{~min}$. Phe, Glu, citrulline, and Gly all decreased compared to baseline. DM4: fifteen AAs (71\%) peaked after $15 \mathrm{~min}$, only five after $30 \mathrm{~min}$, while citrulline peaked after $60 \mathrm{~min}$. All results are displayed in Table 6.

Since most of the AAs peaked after either 15 or $30 \mathrm{~min}$, comparison of the concentrations for each DM after 15 min versus $30 \mathrm{~min}$ was performed to test for significant differences between these time points. The following AAs showed significant changes between time 15 and time 30: DM1: Ala (0.0474), Pro (0.0174), Val (0.0299), and Ile (0.0294), DM2: Leu (0.0295), and DM4: Asp (0.0423). There were no significant changes for any AA in DM3. More details are provided in Supplemental Table A.

The paired $t$-test was used for comparison of the concentrations found in DM1 and DM2 and in DM3 and DM4, respectively, at each time point, to test for significant differences between the four DMs. We found no significant differences at time 0 . Plasma concentrations $(\mu \mathrm{mol} / \mathrm{l})$ after 
TABle 2: Content of pure CGMP (g AA/100 g p) and DM1-4 (pr. $1000 \mathrm{~g}$ mixture) (CGMP and free-synthetic AA). DM1: 100\% CGMP: $158.46 \mathrm{~g}$ = the content of each AA displayed below. DM2: 100\% FSAA (the total amount of AA is shown below). DM3: 119.04 $\mathrm{g}$ from CGMP $(+\mathrm{AA}=$ the additional amount of AA from FSAA). DM4: 100\% FSAA.

\begin{tabular}{|c|c|c|c|c|c|}
\hline CGMP-20 & AA & $\begin{array}{c}\text { DM1: Lacprodan } \\
\text { CGMP-20: } 158.46 \mathrm{~g}^{*}\end{array}$ & $\begin{array}{l}\text { DM2: } \\
\text { FSAA }\end{array}$ & $\begin{array}{c}\text { DM3: Lacprodan } \\
\text { CGMP-20: } 119.04 \mathrm{~g}^{*}+\text { FSAA }\end{array}$ & $\begin{array}{l}\text { DM4: } \\
\text { FSAA }\end{array}$ \\
\hline g AA/100 g protein & \multicolumn{5}{|c|}{ Total amount of AA (g) } \\
\hline 6.4 & Ala & 8.57 & 8.40 & 6.44 & 6.31 \\
\hline 0.3 & Arg & 0.44 & 0.43 & $4.85^{*}$ & 4.85 \\
\hline 9.2 & Asp & 12.08 & 11.83 & 9.07 & 8.89 \\
\hline 0.08 & Cys & 0.15 & 0.15 & 0.11 & 0.11 \\
\hline 21.1 & Glu & 26.53 & 25.99 & 19.93 & 19.52 \\
\hline 1.2 & Gly & 1.53 & 1.50 & 1.15 & 1.13 \\
\hline 0.2 & His & 0.16 & 0.16 & $3.0^{*}$ & 3.04 \\
\hline 11.5 & Ile & 14.50 & 14.21 & 10.89 & 10.67 \\
\hline 2.5 & Leu & 3.12 & 3.06 & $12.11^{*}$ & 12.06 \\
\hline 6.4 & Lys & 8.46 & 8.29 & $7.37^{*}$ & 7.24 \\
\hline 2 & Met & 2.92 & 2.85 & $3.62^{*}$ & 3.57 \\
\hline 0.2 & Phe & 0.20 & 0.20 & 0.15 & 0 \\
\hline 12.6 & Pro & 16.45 & 16.11 & 12.36 & 12.10 \\
\hline 8.5 & Ser & 10.46 & 10.25 & 7.86 & 7.70 \\
\hline 18.1 & Thr & 23.62 & 23.14 & 17.74 & 17.38 \\
\hline 0.04 & $\operatorname{Trp}$ & 0.00 & - & $2.44^{*}$ & 2.44 \\
\hline 0.06 & Tyr & 0.05 & 0.05 & $10.81^{*}$ & 10.81 \\
\hline 9.5 & Val & 11.76 & 11.52 & 8.83 & 8.65 \\
\hline- & Citric acid powder & - & - & 14.40 & 1.40 \\
\hline- & Citric acid solution $(50 \% \mathrm{w} / \mathrm{w}$ in water) & 11.20 & - & - & - \\
\hline- & Tropical twist flavour (IFF SC401962) & 1.75 & 1.75 & 1.75 & 1.80 \\
\hline- & Sucrose & 73.00 & 73.00 & 80.00 & 80.00 \\
\hline- & Water & 755.59 & 787.12 & 751.95 & 780.17 \\
\hline 109.88 & Total & 1000.00 & 1000.00 & 1000.00 & 1000.00 \\
\hline- & $\mathrm{DM}$ & 1 & 2 & 3 & 4 \\
\hline 81 & Protein equivalent $(\mathrm{g} / 100 \mathrm{~g})$ & 12.00 & 12.00 & 12.00 & 12.00 \\
\hline- & Carbohydrate (g/100g) & 7.46 & 7.30 & 8.12 & 8.00 \\
\hline- & Fat $(g / 100 g)$ & 0.03 & 0 & 0.02 & 0 \\
\hline- & Energy (kcal/100 g) & 78 & 77 & 81 & 80 \\
\hline
\end{tabular}

${ }^{*}$ Part of the AA content comes from CGMP and part comes from additional FSAA: Arg: $0.33($ CGMP $)+4.52($ AA $)=4.85$, His $0.12($ CGMP $)+2.92($ AA $)=$ 3.04, Leu: $2.35($ CGMP $)+9.76(\mathrm{AA})=12.11$, Lys: $6.36(\mathrm{CGMP})+1.01(\mathrm{AA})=7.37$, Met: $2.19(\mathrm{CGMP})+1.43(\mathrm{AA})=3.62$, Trp: $0.00(\mathrm{CGMP})+2.44(\mathrm{AA})=2.44$, Tyr: $0.04(\mathrm{CGMP})+10.77(\mathrm{AA})=10.81$.

Table 3: Patient data, all with classic PKU: age, mutations, height, weight, BMI, and usual AA supplement.

\begin{tabular}{lcccccccc}
\hline Patient ID\# & Age & Mutation 1 & Mutation 2 & Phenotype & Height $(\mathrm{cm})$ & Weight $(\mathrm{kg})$ & BMI & Usual AA product \\
\hline 1 & 48 & $\mathrm{c} .1315+1 \mathrm{G}>\mathrm{A}^{1}$ & $\mathrm{c} .1315+1 \mathrm{G}>\mathrm{A}^{1}$ & Classic PKU & 169 & 85 & 30 & PreKUnil tablets \\
2 & 27 & $\mathrm{c} .1315+1 \mathrm{G}>\mathrm{A}^{1}$ & $\mathrm{c} .1222 \mathrm{C}>\mathrm{T}^{2}$ & Classic PKU & 174 & 62 & 20 & XPhe energy \\
3 & 18 & $\mathrm{c} .842 \mathrm{C}>\mathrm{T}^{3}$ & $\mathrm{c} .1315+1 \mathrm{G}>\mathrm{A}^{1}$ & Classic PKU & 171 & 75 & 26 & Avonil powder \\
4 & 16 & $\mathrm{c} .1222 \mathrm{C}>\mathrm{T}^{2}$ & $\mathrm{c} .1315+1 \mathrm{G}>\mathrm{A}^{1}$ & Classic PKU & 171 & 47 & 16 & Avonil tablets \\
5 & 38 & $\mathrm{c} .1315+1 \mathrm{G}>\mathrm{A}^{1}$ & $\mathrm{c} .1222 \mathrm{C}>\mathrm{T}^{2}$ & Classic PKU & 179 & 79 & 25 & PreKUnil tablets \\
6 & 46 & $\mathrm{c} .814 \mathrm{G}>\mathrm{T}^{4}$ & $\mathrm{c} .1222 \mathrm{C}>\mathrm{T}^{2}$ & Classic PKU & 173 & 94 & 31 & PreKUnil tablets \\
7 & 34 & $\mathrm{c} .473 \mathrm{G}>\mathrm{A}^{5}$ & $\mathrm{c} .1315+1 \mathrm{G}>\mathrm{A}^{1}$ & Classic PKU & 162 & 53 & 20 & PreKUnil tablets \\
8 & 39 & $\mathrm{c} .1222 \mathrm{C}>\mathrm{T}^{2}$ & $\mathrm{c} .1222 \mathrm{C}>\mathrm{T}^{2}$ & Classic PKU & 162 & 87.5 & 33 & Avonil tablets \\
\hline 1 & & & & & & & &
\end{tabular}

${ }^{1}(\mathrm{IVS} 12+1 \mathrm{G}>\mathrm{A}) ;{ }^{2}$ p.R408W; ${ }^{3}$ p.E280K; ${ }^{4}$ p.G272X; ${ }^{5}$ p.R158Q. 
TABLE 4: Intake of DM (water + CGMP mixture powder = total volume) and standard meal in grams (g) and energy (kilojoule (kJ) and energy \% (E \%)).

\begin{tabular}{|c|c|c|c|c|c|c|c|c|c|c|}
\hline Patient ID & 1 & 2 & 3 & 4 & 5 & 6 & 7 & 8 & Mean & $\mathrm{SD}$ \\
\hline Powder (g) & 41 & 30 & 36 & 21 & 38 & 45 & 27 & 44 & 35 & 8 \\
\hline Water (g) & 136 & 99 & 120 & 77 & 126 & 151 & 83 & 139 & 116 & 25 \\
\hline LP bread (g) & 70 & 90 & 170 & 100 & 82 & 70 & 96 & 80 & 95 & 30 \\
\hline Butter (g) & 23 & 23 & 35 & 26 & 21 & 21 & 23 & 19 & 24 & 5 \\
\hline Marmalade (g) & 40 & 40 & 40 & 42 & 40 & 40 & 40 & 40 & 40 & 1 \\
\hline Energy (kJ) & 2324 & 2330 & 3445 & 2405 & 2324 & 2327 & 2316 & 2322 & 2474 & 368 \\
\hline Protein (g) & 22 & 16 & 20 & 13 & 20 & 24 & 14 & 23 & 19 & 4 \\
\hline Protein E (\%) & 16 & 12 & 10 & 9 & 15 & 18 & 10 & 17 & 13 & 3 \\
\hline Fat $(g)$ & 20 & 20 & 32 & 23 & 19 & 18 & 21 & 18 & 21 & 4 \\
\hline Fat E (\%) & 32 & 33 & 34 & 35 & 30 & 29 & 33 & 28 & 32 & 2 \\
\hline Carbohydrate (g) & 70 & 75 & 111 & 78 & 74 & 71 & 76 & 74 & 79 & 12 \\
\hline Carbohydrate E (\%) & 52 & 56 & 56 & 57 & 55 & 53 & 57 & 56 & 55 & 2 \\
\hline
\end{tabular}

TABLE 5: Results for the AUC ( $\mu \mathrm{mol} / \mathrm{l}$ over time) for all AAs (adjusted for baseline (time 0$)$ ). Significant differences for the following AAs for the area under the curve (AUC) minus baseline: DM1 and 2: Lys $(p=0.0287)$, Asn $(p=0.0201)$, and Asp $(p=0.0046)$ and DM3 and 4: citrulline $(p=0.0162)$.

\begin{tabular}{|c|c|c|c|c|c|c|c|c|}
\hline \multirow{2}{*}{$\mathrm{DM}$} & \multicolumn{2}{|c|}{1} & \multicolumn{2}{|c|}{2} & \multicolumn{2}{|c|}{3} & \multicolumn{2}{|c|}{4} \\
\hline & Mean & SD & Mean & SD & Mean & SD & Mean & SD \\
\hline Gly & -1145 & 12734 & 11361 & 20714 & -16590 & 15386 & 11709 & 9483 \\
\hline Ala & 38797 & 10277 & 29471 & 5292 & 8397 & 3693 & 20653 & 5239 \\
\hline Ser & 8021 & 2312 & 6262 & 680 & -1264 & 2222 & 2180 & 1230 \\
\hline Pro & 28232 & 3802 & 33492 & 7623 & 9353 & 2444 & 15950 & 2204 \\
\hline Val & 46664 & 5818 & 33878 & 7511 & 14370 & 5722 & 24624 & 7244 \\
\hline Thr & 38627 & 7843 & 35865 & 3144 & 13558 & 1338 & 20698 & 2662 \\
\hline Leu & -3102 & 499 & -2979 & 786 & 6899 & 1798 & 5206 & 1564 \\
\hline Ile & 26638 & 2253 & 21551 & 1691 & 9666 & 1474 & 8429 & 1146 \\
\hline Lys & 16623 & 3356 & 5005 & 3255 & 3932 & 2076 & 7342 & 2542 \\
\hline Asn & 3036 & 688 & 910 & 409 & 1838 & 978 & 1129 & 652 \\
\hline Met & 1976 & 580 & 1848 & 230 & 842 & 429 & 3026 & 1961 \\
\hline His & -419 & 768 & -1133 & 677 & -1036 & 1151 & 1003 & 697 \\
\hline Phe & -6717 & 11135 & -2778 & 6468 & -24356 & 10869 & -8944 & 9170 \\
\hline Tyr & -3376 & 592 & -4000 & 649 & 6672 & 1012 & 6019 & 1622 \\
\hline Glu & 123 & 399 & -305 & 541 & -2302 & 1004 & -988 & 782 \\
\hline Gln & 34247 & 7716 & 39614 & 10878 & -4902 & 4414 & 9870 & 7528 \\
\hline Asp & 1399 & 413 & -254 & 239 & -682 & 490 & -469 & 217 \\
\hline $\operatorname{Trp}$ & -1364 & 403 & -1078 & 412 & 804 & 849 & 994 & 484 \\
\hline Orn & 1167 & 818 & 733 & 621 & 799 & 724 & 1288 & 304 \\
\hline Arg & -505 & 1292 & -100 & 1136 & -1121 & 1310 & 1047 & 941 \\
\hline Cit & -1115 & 634 & 790 & 647 & -2193 & 549 & 305 & 652 \\
\hline
\end{tabular}

intake of the DM and test meal for all AAs presented as \%. Comparison between $\mathrm{DM} 1+2$ and $\mathrm{DM} 3+4$ gave the following results: time 15: DM1+2: Tyr: 0.0228, Asp: 0.0136, and citrulline: 0.0378; DM3+4: Pro: 0.0455, Val: 0.0204, and Thr: 0.0239; time 30: DM1+2: Leu: 0.0178, Ile: 0.0021, Asn: 0.0038, and Asp: 0.0362; DM3+4: Ser: 0.0031, Pro: 0.0486, Thr: 0.0027, His: 0.0108, Tyr: 0.0412, and citrulline: 0.0409; time 60: DM1+2: Asp: 0.0060 and citrulline: 0.0100; DM3+4: Pro: 0.0003, Val: 0.0088, Thr: 0.0270, and Asp: 0.0155; time
120: DM3+4: citrulline:0.0364; and time 240: DM1+2: Asp: 0.0288 . There were no significant differences at time 0 .

Compared to baseline values, ghrelin values demonstrated a significant decrease at 30,60, and 120 min for DM1, at $30 \mathrm{~min}$ for DM2, at 60 and $120 \mathrm{~min}$ for DM3, and at 30, 60, and 120 min for DM4, respectively. Final levels decreased 7\% for DM1, increased $10 \%$ for DM2 and 5\% for DM3, and remained unchanged in DM4, all compared to baseline. Table 7 displays results (\% compared to baseline) and the 
TABle 6: Plasma concentrations $(\mu \mathrm{mol} / \mathrm{l})$ after intake of the DM and test meal for all AAs presented as \% compared to time 0 (=1). Comparison between DM1+2 and DM3+4 gave the following results: time 15: DM1+2: Tyr: 0.0228, Asp: 0.0136, citrulline: 0.0378; DM3+4: Pro: 0.0455, Val: 0.0204, Thr: 0.0239; time 30: DM1+2: Leu: 0.0178, Ile: 0.0021, Asn: 0.0038, Asp: 0.0362; DM3+4: Ser: 0.0031, Pro: 0.0486, Thr: 0.0027, His: 0.0108, Tyr: 0.0412, citrulline: 0.0409; time 60: DM1+2: Asp: 0.0060, citrulline: 0.0100; DM3+4: Pro: 0.0003, Val: 0.0088, Thr: 0.0270, Asp: 0.0155; time 120: DM3+4: citrulline: 0.0364; time 240: DM1+2: Asp: 0.0288. There were no significant differences at time 0.

\begin{tabular}{|c|c|c|c|c|c|c|c|c|c|c|}
\hline \multirow{2}{*}{ Time } & \multicolumn{2}{|c|}{15} & \multicolumn{2}{|c|}{30} & \multicolumn{2}{|c|}{60} & \multicolumn{2}{|c|}{120} & \multicolumn{2}{|c|}{240} \\
\hline & Mean & $\mathrm{SD}$ & Mean & $\mathrm{SD}$ & Mean & $\mathrm{SD}$ & Mean & SD & Mean & SD \\
\hline & & & & & DM1 & & & & & \\
\hline Gly & 0.961 & 0.339 & 1.062 & 0.616 & 0.944 & 0.500 & 1.011 & 0.355 & 0.963 & 0.717 \\
\hline Ala & 1.656 & 0.435 & 2.340 & 0.653 & 1.940 & 0.462 & 1.510 & 0.419 & 1.233 & 0.328 \\
\hline Ser & 1.669 & 0.434 & 2.189 & 0.615 & 1.535 & 0.434 & 1.024 & 0.172 & 1.194 & 0.440 \\
\hline Pro & 2.904 & 0.886 & 3.886 & 1.117 & 2.819 & 1.256 & 1.782 & 0.303 & 1.523 & 0.374 \\
\hline Val & 1.977 & 0.510 & 2.509 & 0.698 & 2.255 & 0.422 & 1.712 & 0.257 & 1.583 & 0.310 \\
\hline Thr & 2.594 & 0.544 & 3.885 & 0.867 & 2.855 & 0.813 & 2.013 & 0.229 & 1.832 & 0.829 \\
\hline Leu & 1.365 & 0.152 & 1.425 & 0.155 & 0.995 & 0.094 & 0.582 & 0.162 & 0.828 & 0.143 \\
\hline Ile & 5.815 & 1.849 & 7.033 & 1.190 & 5.198 & 0.841 & 3.426 & 0.464 & 2.603 & 0.517 \\
\hline Lys & 2.195 & 0.794 & 2.258 & 0.500 & 1.828 & 0.690 & 1.468 & 0.653 & 1.263 & 0.734 \\
\hline Asn & 1.666 & 0.251 & 2.003 & 0.399 & 1.412 & 0.313 & 1.012 & 0.091 & 1.218 & 0.414 \\
\hline Met & 2.255 & 0.714 & 2.275 & 0.684 & 1.690 & 0.522 & 1.165 & 0.235 & 1.071 & 0.387 \\
\hline His & 1.000 & 0.116 & 1.153 & 0.245 & 1.034 & 0.231 & 0.842 & 0.118 & 1.060 & 0.339 \\
\hline Phe & 0.942 & 0.130 & 1.012 & 0.189 & 0.990 & 0.199 & 0.935 & 0.131 & 0.976 & 0.271 \\
\hline Tyr & 0.816 & 0.143 & 0.863 & 0.245 & 0.765 & 0.170 & 0.599 & 0.134 & 0.647 & 0.136 \\
\hline Glu & 1.302 & 0.307 & 1.243 & 0.149 & 1.061 & 0.180 & 0.915 & 0.127 & 0.961 & 0.173 \\
\hline Gln & 1.278 & 0.204 & 1.309 & 0.296 & 1.291 & 0.277 & 1.245 & 0.208 & 1.242 & 0.402 \\
\hline Asp & 1.783 & 0.495 & 2.225 & 0.760 & 1.592 & 0.327 & 1.076 & 0.245 & 1.104 & 0.316 \\
\hline $\operatorname{Trp}$ & 0.980 & 0.105 & 1.116 & 0.188 & 0.906 & 0.155 & 0.725 & 0.145 & 0.808 & 0.256 \\
\hline Orn & 1.288 & 0.340 & 1.520 & 0.501 & 1.287 & 0.366 & 0.989 & 0.175 & 1.006 & 0.325 \\
\hline Arg & 1.041 & 0.135 & 1.220 & 0.272 & 1.024 & 0.198 & 0.876 & 0.193 & 0.963 & 0.242 \\
\hline Cit & 0.889 & 0.251 & 1.075 & 0.316 & 0.809 & 0.228 & 0.744 & 0.244 & 0.939 & 0.467 \\
\hline & & & & & DM2 & & & & & \\
\hline Gly & 1.238 & 0.926 & 1.197 & 0.745 & 1.081 & 0.938 & 1.227 & 0.796 & 0.979 & 0.654 \\
\hline Ala & 1.603 & 0.354 & 1.664 & 0.483 & 1.620 & 0.447 & 1.312 & 0.335 & 1.163 & 0.379 \\
\hline Ser & 1.653 & 0.297 & 1.697 & 0.546 & 1.457 & 0.204 & 1.102 & 0.213 & 1.083 & 0.169 \\
\hline Pro & 3.177 & 0.867 & 3.052 & 1.017 & 2.846 & 1.185 & 2.291 & 1.110 & 1.617 & 0.435 \\
\hline Val & 1.647 & 0.579 & 1.856 & 0.730 & 1.719 & 0.397 & 1.544 & 0.560 & 1.420 & 0.497 \\
\hline Thr & 2.555 & 0.512 & 3.182 & 0.977 & 2.808 & 0.691 & 2.254 & 0.735 & 1.957 & 0.447 \\
\hline Leu & 1.389 & 0.255 & 1.225 & 0.196 & 0.983 & 0.094 & 0.635 & 0.129 & 0.810 & 0.160 \\
\hline Ile & 4.892 & 2.668 & 4.640 & 2.055 & 4.192 & 1.679 & 3.112 & 1.420 & 2.572 & 0.857 \\
\hline Lys & 1.714 & 0.725 & 1.420 & 0.530 & 1.280 & 0.341 & 1.046 & 0.218 & 1.023 & 0.438 \\
\hline Asn & 1.518 & 0.172 & 1.314 & 0.359 & 1.155 & 0.124 & 0.954 & 0.197 & 1.046 & 0.270 \\
\hline Met & 1.992 & 0.542 & 1.830 & 0.641 & 1.570 & 0.328 & 1.220 & 0.173 & 1.085 & 0.184 \\
\hline His & 1.114 & 0.208 & 1.045 & 0.308 & 0.993 & 0.175 & 0.823 & 0.178 & 0.944 & 0.171 \\
\hline Phe & 1.055 & 0.227 & 0.952 & 0.195 & 0.956 & 0.109 & 0.964 & 0.159 & 1.036 & 0.130 \\
\hline Tyr & 0.951 & 0.231 & 0.812 & 0.187 & 0.692 & 0.132 & 0.586 & 0.145 & 0.606 & 0.125 \\
\hline Glu & 1.130 & 0.182 & 1.087 & 0.177 & 1.016 & 0.165 & 0.897 & 0.222 & 0.964 & 0.202 \\
\hline Gln & 1.537 & 0.427 & 1.508 & 0.350 & 1.478 & 0.531 & 1.199 & 0.393 & 1.271 & 0.390 \\
\hline Asp & 1.200 & 0.426 & 1.132 & 0.266 & 0.880 & 0.155 & 0.814 & 0.230 & 1.005 & 0.190 \\
\hline $\operatorname{Trp}$ & 1.075 & 0.304 & 0.996 & 0.361 & 0.871 & 0.263 & 0.768 & 0.171 & 0.884 & 0.171 \\
\hline Orn & 1.227 & 0.290 & 1.081 & 0.287 & 1.067 & 0.211 & 1.084 & 0.302 & 1.079 & 0.275 \\
\hline Arg & 1.207 & 0.286 & 1.111 & 0.395 & 1.084 & 0.215 & 0.927 & 0.215 & 0.931 & 0.258 \\
\hline Cit & 1.118 & 0.525 & 1.136 & 0.404 & 1.211 & 0.349 & 1.121 & 0.359 & 1.030 & 0.359 \\
\hline
\end{tabular}


TABle 6: Continued.

\begin{tabular}{|c|c|c|c|c|c|c|c|c|c|c|}
\hline \multirow{2}{*}{ Time } & \multicolumn{2}{|c|}{15} & \multicolumn{2}{|c|}{30} & \multicolumn{2}{|c|}{60} & \multicolumn{2}{|c|}{120} & \multicolumn{2}{|c|}{240} \\
\hline & Mean & SD & Mean & SD & Mean & SD & Mean & SD & Mean & SD \\
\hline & & & & & DM3 & & & & & \\
\hline Gly & 0.809 & 0.355 & 0.913 & 0.337 & 0.822 & 0.621 & 0.772 & 0.318 & 0.804 & 0.679 \\
\hline Ala & 1.206 & 0.304 & 1.435 & 0.302 & 1.171 & 0.192 & 1.085 & 0.277 & 0.987 & 0.141 \\
\hline Ser & 1.210 & 0.211 & 1.248 & 0.262 & 0.941 & 0.194 & 0.862 & 0.292 & 0.900 & 0.200 \\
\hline Pro & 1.777 & 0.313 & 1.838 & 0.349 & 1.471 & 0.234 & 1.278 & 0.415 & 1.126 & 0.242 \\
\hline Val & 1.459 & 0.274 & 1.715 & 0.330 & 1.228 & 0.117 & 1.314 & 0.572 & 1.078 & 0.143 \\
\hline Thr & 1.625 & 0.368 & 1.851 & 0.471 & 1.635 & 0.217 & 1.438 & 0.463 & 1.325 & 0.293 \\
\hline Leu & 2.282 & 0.435 & 2.328 & 0.442 & 1.658 & 0.366 & 1.091 & 0.617 & 0.887 & 0.141 \\
\hline Ile & 3.524 & 1.089 & 3.675 & 0.890 & 2.498 & 0.721 & 1.736 & 1.234 & 1.380 & 0.386 \\
\hline Lys & 1.632 & 0.560 & 1.662 & 0.712 & 1.389 & 0.599 & 0.943 & 0.117 & 0.874 & 0.189 \\
\hline Asn & 1.630 & 0.329 & 1.534 & 0.429 & 1.353 & 0.335 & 1.034 & 0.430 & 0.980 & 0.202 \\
\hline Met & 1.826 & 0.370 & 1.754 & 0.507 & 1.255 & 0.116 & 1.070 & 0.374 & 0.865 & 0.180 \\
\hline His & 1.049 & 0.153 & 1.153 & 0.240 & 0.915 & 0.137 & 0.900 & 0.264 & 0.895 & 0.129 \\
\hline Phe & 0.889 & 0.111 & 0.911 & 0.104 & 0.843 & 0.100 & 0.879 & 0.140 & 0.910 & 0.119 \\
\hline Tyr & 1.645 & 0.244 & 1.985 & 0.713 & 1.723 & 0.437 & 1.667 & 0.295 & 1.305 & 0.248 \\
\hline Glu & 0.950 & 0.144 & 0.951 & 0.152 & 0.801 & 0.189 & 0.756 & 0.221 & 0.774 & 0.111 \\
\hline Gln & 1.170 & 0.104 & 1.061 & 0.223 & 1.036 & 0.120 & 0.911 & 0.148 & 0.920 & 0.093 \\
\hline Asp & 0.953 & 0.291 & 1.111 & 0.138 & 0.998 & 0.158 & 0.755 & 0.221 & 0.724 & 0.170 \\
\hline $\operatorname{Trp}$ & 1.244 & 0.272 & 1.455 & 0.302 & 1.165 & 0.382 & 1.071 & 0.444 & 0.950 & 0.383 \\
\hline Orn & 1.259 & 0.247 & 1.374 & 0.230 & 1.147 & 0.222 & 1.064 & 0.335 & 0.977 & 0.195 \\
\hline Arg & 1.216 & 0.226 & 1.236 & 0.331 & 0.982 & 0.138 & 0.869 & 0.213 & 0.835 & 0.163 \\
\hline Cit & 0.815 & 0.158 & 0.802 & 0.173 & 0.649 & 0.163 & 0.642 & 0.141 & 0.842 & 0.211 \\
\hline & & & & & DM4 & & & & & \\
\hline Gly & 1.179 & 0.781 & 1.304 & 0.630 & 0.920 & 0.445 & 1.230 & 0.433 & 1.183 & 0.690 \\
\hline Ala & 1.546 & 0.395 & 1.552 & 0.278 & 1.463 & 0.406 & 1.304 & 0.157 & 1.003 & 0.378 \\
\hline Ser & 1.521 & 0.336 & 1.419 & 0.228 & 1.196 & 0.382 & 0.978 & 0.120 & 0.961 & 0.347 \\
\hline Pro & 2.499 & 1.154 & 2.357 & 0.774 & 2.003 & 0.704 & 1.509 & 0.287 & 1.135 & 0.429 \\
\hline Val & 1.861 & 0.438 & 1.865 & 0.450 & 1.528 & 0.297 & 1.511 & 0.913 & 1.263 & 0.491 \\
\hline Thr & 2.067 & 1.025 & 2.389 & 0.795 & 2.069 & 0.687 & 1.654 & 0.224 & 1.380 & 0.525 \\
\hline Leu & 2.237 & 0.667 & 1.981 & 0.433 & 1.579 & 0.482 & 0.995 & 0.277 & 0.829 & 0.279 \\
\hline Ile & 3.681 & 1.813 & 3.072 & 1.203 & 2.667 & 1.251 & 1.481 & 0.582 & 1.132 & 0.457 \\
\hline Lys & 1.671 & 0.782 & 1.385 & 0.292 & 1.429 & 0.704 & 1.184 & 0.389 & 1.179 & 0.589 \\
\hline Asn & 1.588 & 0.591 & 1.502 & 0.439 & 1.291 & 0.342 & 1.008 & 0.191 & 0.851 & 0.231 \\
\hline Met & 2.527 & 1.706 & 2.337 & 1.887 & 1.956 & 2.094 & 1.503 & 1.681 & 1.035 & 0.763 \\
\hline His & 1.237 & 0.231 & 1.261 & 0.135 & 1.140 & 0.270 & 1.004 & 0.153 & 0.981 & 0.342 \\
\hline Phe & 1.054 & 0.126 & 0.981 & 0.109 & 0.947 & 0.141 & 0.942 & 0.108 & 0.921 & 0.293 \\
\hline Tyr & 1.548 & 0.594 & 1.524 & 0.559 & 1.606 & 0.723 & 1.667 & 0.568 & 1.347 & 0.525 \\
\hline Glu & 1.123 & 0.205 & 1.013 & 0.191 & 0.950 & 0.221 & 0.823 & 0.168 & 0.893 & 0.407 \\
\hline Gln & 1.236 & 0.246 & 1.150 & 0.160 & 1.120 & 0.211 & 1.107 & 0.196 & 0.911 & 0.319 \\
\hline Asp & 1.121 & 0.261 & 0.964 & 0.149 & 0.854 & 0.234 & 0.819 & 0.177 & 0.860 & 0.343 \\
\hline $\operatorname{Trp}$ & 1.505 & 0.436 & 1.403 & 0.272 & 1.252 & 0.345 & 1.031 & 0.147 & 1.010 & 0.355 \\
\hline Orn & 1.470 & 0.405 & 1.376 & 0.200 & 1.337 & 0.302 & 1.105 & 0.153 & 0.992 & 0.314 \\
\hline Arg & 1.477 & 0.315 & 1.344 & 0.238 & 1.138 & 0.218 & 0.967 & 0.116 & 0.940 & 0.314 \\
\hline Cit & 1.132 & 0.285 & 1.105 & 0.216 & 1.201 & 0.603 & 0.989 & 0.231 & 0.971 & 0.413 \\
\hline
\end{tabular}

$t$-test for all levels and values (mean and SD) in Supplemental Table B.

None of the biomarkers GLP-1, PYY, BUN, CCK, insulin, and glucose showed significant changes from baseline (premeal) to the end of the study period (240 min after meal and DM). Results for biomarkers are presented in Figure 1 (\% compared to baseline) and values (mean +/- SD) in Supplemental Table C. 
TABLE 7: Ghrelin levels over time, presented as \% relative to start value (time 0$)+\mathrm{SD}$ and $p$ value.

\begin{tabular}{|c|c|c|c|c|c|c|c|c|c|c|c|c|}
\hline \multirow[b]{2}{*}{ Time } & \multicolumn{3}{|c|}{ DM1 } & \multicolumn{3}{|c|}{ DM2 } & \multicolumn{3}{|c|}{ DM3 } & \multicolumn{3}{|c|}{ DM4 } \\
\hline & $\begin{array}{c}\text { Relative } \\
(\%)\end{array}$ & $\begin{array}{l}\text { SD } \\
(\%)\end{array}$ & $p$ value & $\begin{array}{c}\text { Relative } \\
(\%)\end{array}$ & $\begin{array}{l}\text { SD } \\
(\%)\end{array}$ & $p$ value & $\begin{array}{c}\text { Relative } \\
(\%)\end{array}$ & $\begin{array}{l}\mathrm{SD} \\
(\%)\end{array}$ & $p$ value & $\begin{array}{c}\text { Relative } \\
(\%)\end{array}$ & $\begin{array}{l}\mathrm{SD} \\
(\%)\end{array}$ & $p$ value \\
\hline 0 & 100 & - & - & 100 & - & - & 100 & - & - & 100 & - & - \\
\hline 15 & 95 & 12 & 0.266 & 88 & 11 & 0.056 & 90 & 13 & 0.171 & 75 & 22 & 0.050 \\
\hline 30 & 82 & 5 & $0.006^{*}$ & 83 & 15 & $0.035^{*}$ & 78 & 19 & 0.055 & 80 & 10 & $0.015^{*}$ \\
\hline 60 & 81 & 13 & $0.030^{*}$ & 84 & 16 & 0.068 & 80 & 12 & $0.013^{*}$ & 75 & 11 & $0.006^{*}$ \\
\hline 120 & 81 & 10 & $0.020^{*}$ & 84 & 15 & 0.088 & 75 & 14 & $0.011^{*}$ & 74 & 13 & $0.010^{*}$ \\
\hline 240 & 93 & 18 & 0.454 & 110 & 20 & 0.180 & 105 & 25 & 0.473 & 100 & 34 & 0.986 \\
\hline
\end{tabular}

*Significant.

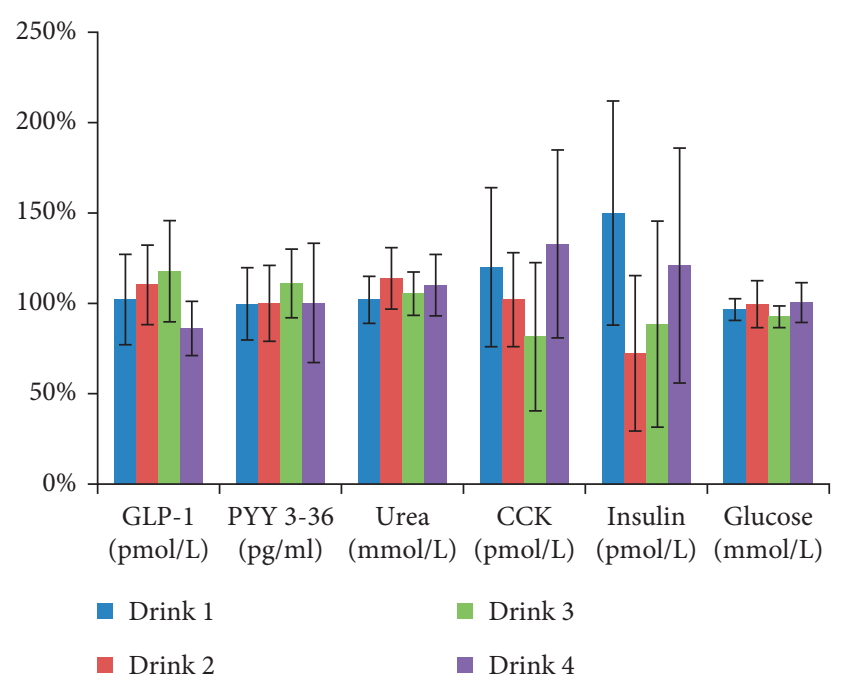

Figure 1: Results (mean $+/-\mathrm{SD}$ ) for the following biomarkers: glucose, insulin, GLP-1, PYY, BUN, and CCK. None of them demonstrated significant changes from baseline (premeal) to the end of the study period ( 240 min after meal and DM).

3.6. VAS Score. The question "how satisfied are you?" obtained the following nonsignificant results: DM1 (mean 36, SD 18), DM2 (mean 41, SD 16), DM3 (mean 28, SD 27), and DM4 (mean 35, SD 30). The results for the other question "how does the DM taste?" were as follows: DM1 (mean 46, SD 31), DM2 (mean 44, SD 22), DM3 (mean 36, SD 28), and DM4 (mean 26, SD 22). All comparisons (DM1 and DM2, DM3 and DM4, but also DM3 compared to DM1 and DM2, resp.) were statistically insignificant.

\section{Discussion}

This study evaluated the metabolic short-term effect of CGMP compared to an almost identical combination of FSAA by repeated measurements. One of the most important findings was that the residual content of Phe in DM3 did not affect the plasma level significantly compared to DM4 at any time which support data from Ney et al. [11].

Over the last 8 years, CGMP has been investigated in mice studies and a few human studies to evaluate safety, acceptability, and efficacy of CGMP as a nutritional supplement for treatment of PKU [11-13, 15, 30, 31]. The present study supports the conclusions of these studies.

The slower absorption of most of the AA in DM1 and DM3, which contained CGMP, compared to DM2 and DM4, which contained only FSAA, could be explained by the fact that CGMP delay the absorption in the gut. The fact that Tyr increased in DM3 and DM4, but only decreased without peak in DM1 and DM2, is assumed to be caused by the low content in DM1 and DM2. Tyr and Trp both peaked at $30 \mathrm{~min}$ for DM3 compared to $15 \mathrm{~min}$ for DM4, suggesting that the content of Tyr and Trp in the CGMP mixtures were metabolized less rapidly than the FSAA. The Phe/Tyr ratio decreased over time with $30 \%$ in both DM3 and DM4, while it increased (caused by the low-Tyr concentration in DM1 and DM2) with $50 \%$ for DM1 and $70 \%$ for DM2. The Phe/Tyr ratio is an important measure because a high ratio can have a long-term negative effect on executive functions [32].

The AUC for "total AA" was not associated with absorption rate. We also calculated the AUC for each AA and compared with peak values to determine complete absorption and absorption rate, and we did find significant differences for Lys, Asn, and Asp for DM1 and DM2 and for citrulline for DM3 and DM4. None of the LNAA (extraadded in DM3 and matched in DM4) was significantly different.

Ala, Pro, Val, and Ile demonstrated a significant increase from 15 to 30 min for DM1, while only Leu in DM2 and Asp in DM4 decreased significantly, which indicate a better absorption of pure CGMP. The fact that none of the AA in DM3 changed significantly could be the influence of the extra-added AA (LNAA and Lys). In contrast, we did see significant differences between several AAs by comparison between DM1 and DM2 and between DM3 and DM4 at each time point. Especially His, Tyr, and Trp are noteworthy for DM3 and DM4, since they are FSAAs added to the pure CGMP. Also, Thr, Ile, and Val are of certain interest, since the natural content of these three AAs in CGMP is high [33]. The high content of these AAs and addition of extra-LNAA to the CGMP offer an additional positive effect, since LNAA competes for transport across the blood-brain barrier (BBB) via the L-type amino acid transporter (LAT1) [34, 35]. High Phe in plasma diminishes uptake of Tyr and Trp into the brain, and this results in reduced formation of neurotransmitters [36]. This imbalance is possibly the major cause of disturbed brain development in PKU patients [37]. Each 
LNAA has individual affinity relation to LAT1 [38]. Several studies have shown the positive blocking effect of LNAA which reduces Phe entering the brain [39-41]. Matalon et al. [17] demonstrated a decrease in Phe in the blood up to $50 \%$ using LNAA tablets and emphasized that LNAA in a specific mixture inhibits Phe uptake already in the gut $[16,42]$. Administrations of Val, Ile, and Leu have proved to reduce Phe concentrations in the cerebrospinal fluid of humans [43].

All biomarkers remained unchanged by comparing time 0 and $240 \mathrm{~min}$, and there were no significant changes in plasma Phe despite the residual amount of Phe in CGMP in line with findings from Ney et al. [11, 13]. This study demonstrated that AA in CGMP is absorbed as efficient as an identical mixture of FSAA.

All the DMs demonstrated a decreasing effect on ghrelin after the meal. Ghrelin values showed significant decreases at 30,60, and $120 \mathrm{~min}$ for DM1, at $30 \mathrm{~min}$ for DM2, at 60 and $120 \mathrm{~min}$ for DM3, and at 30, 60, and $120 \mathrm{~min}$ for DM4. Low-ghrelin concentrations are associated with a feeling of satiety $[44,45]$. By only evaluating DM1 and DM2, it could indicate that satiety is reached faster for CGMP. However, VAS scores for satiety did not show any significant difference between DM1 and DM2, nor between DM3 and DM4.

BUN was nonsignificantly lower for DM1 and DM3 compared to DM2 and DM4, which potentially could suggest a more efficient utilization of GMP compared to AA, as found by van Calcar et al. [13]. Similar findings have been reported by Ney et al. [46]. However, our present short-term study was not able to support these findings.

GLP-1 promotes insulin secretion and reduces appetite and reached the highest (nonsignificant) level with DM3 after $240 \mathrm{~min}(118 \%)$ which may indicate that satiety was better obtained with DM3 compared with DM1, DM2, and DM4. This finding concurs with previous studies, showing that GMP promotes satiety [14]. PYY also reduces appetite and reached the highest value for DM3 (111\%). CCK is a peptide hormone in the gastrointestinal system responsible for stimulating the digestion of fat and protein. The wide variation from a decrease of $18 \%$ in DM3 to an increase of $33 \%$ in DM4 was unexpected, since CCK is expected to rise after a mixed meal [23].

A limitation of this study was the small number of patients. However, it was important for the study design to select as homogeneous a test population as possible (only early-treated classical PKU confirmed by mutation analysis), resulting in exclusion of a number of patients.

Three patients had a BMI over 30 and were defined as obese, one slightly obese, three had a normal BMI, and one had a BMI below normal. The patients were receiving dosage after their actual weight, which means that the patients that were categorized with normal BMI received less DM per $\mathrm{kg}$ lean body mass.

Although this study demonstrated nonsignificant changes for almost all biochemical markers, it is important to notice that this study only replaced a single meal and a long-term effect could be different. Based on the current results, we consider CGMP to be a safe alternative to FSAA but should be supplemented with additional FSAA to make it nutritionally adequate and potential also with other nutrients as fat, carbohydrates, vitamins, and minerals to create an easy-to-use supplement for patients with PKU. If CGMP products substitute FSAA completely, it is necessary to carefully monitor if the small content of Phe will have an impact on the blood level in the long term. If Phe levels increase, restrictions of the LP diet must be implemented to balance and control the Phe intake and blood level. Further studies are needed to evaluate the long-term impact and efficacy of CGMP in the management of PKU.

\section{Conclusion}

Dietary management of PKU should be lifelong, and good compliance is crucial for a good outcome. CGMP did not change any of the biomarkers significantly compared to freesynthetic AA when consumed with a test meal in PKU patients. The residual amount of Phe in CGMP did not affect the plasma Phe level significantly. Based on these data, we consider CGMP to be a suitable alternative as supplement for PKU treatment. However, further research is needed to determine the long-term effects and safety of CGMP. This study demonstrates that CGMP has the same short-term effect as FSAA.

\section{Abbreviations}

CGMP: Casein glycomacropeptide, CGMP-20 (product name Lacprodan CGMP-20)

PKU: Phenylketonuria

AA: Amino acid(s)

FSAA: Free-synthetic AA

BBB: Blood-brain barrier

BUN: Blood urea nitrogen

GLP-1: Glucagon-like peptide-1

CCK: Cholecystokinin

PYY: Peptide tyrosine-tyrosine

DM: Drink mix

Ala: Alanine

Arg: Arginine

Asn: Asparagine

Asp: Aspartate

Cys: Cysteine

Glu: Glutamine

Gly: Glycine

His: Histidine

Ile: Isoleucine

Leu: Leucine

Lys: Lysine

Met: Methionine

Phe: Phenylalanine

Pro: Proline

Ser: $\quad$ Serine

Thr: Threonine

Trp: Tryptophan

Tyr: Tyrosine

Val: Valine

PAH: Phenylalanine hydroxylase

VAS: Visual analogue score 
LP: $\quad$ Low protein

min: $\quad$ Minutes

SD: $\quad$ Standard deviation

kJ: Kilojoule.

\section{Disclosure}

Kirsten Kiær Ahring is an industrial PhD student sponsored by AFI and The Danish Agency for Science, Technology and Innovation.

\section{Conflicts of Interest}

The authors declare that they have no conflicts of interest.

\section{Authors' Contributions}

All authors have made a substantial contribution to the design and interpretation of data for the work, revised it critically, and been presented for the final version to be published. Erik Jensen, Kirsten K. Ahring, and Lisbeth B. Møller were involved in designing the study. Kirsten K. Ahring was involved in collecting all the data and writing the main manuscript.

\section{Acknowledgments}

The authors would like to thank Arla Foods Ingredients and The Danish Agency for Science, Technology and Innovation for funding, the PKU patients for participating in the study, nurse Signe Larsen (PKU clinic, Kennedy Centre) for performing the blood sampling, and lab technicians Søren Andresen and Marianne Falck (University of Copenhagen) for performing the blood analyses and assisting in handling of blood samples.

\section{Supplementary Materials}

Table A. Results for all AA at time 0, 15, 30, 60, 120, and 240 (Mean and SD). Table B. Ghrelin results for time 0, 15, 30, 60, 120, and 240 (Mean and SD). Table C. Results for glucose, insulin, GLP-1, PYY, BUN, and CCK (Mean \pm SD). None of them demonstrated significant changes from baseline (premeal) to end of study period (240 min after meal and DM). (Supplementary Materials)

\section{References}

[1] J. J. Mitchell, Y. J. Trakadis, and C. R. Scriver, "Phenylalanine hydroxylase deficiency," Genetics in Medicine, vol. 13, no. 8, pp. 697-707, 2011.

[2] N. Blau, "Genetics of phenylketonuria: then and now," Human Mutation, vol. 37, no. 6, pp. 508-515, 2016.

[3] M. Giovannini, E. Verduci, E. Salvatici, S. Paci, and E. Riva, "Phenylketonuria: nutritional advances and challenges," Nutrition and Metabolism, vol. 9, no. 1, p. 7, 2012.

[4] H. L. Levy, "Nutritional therapy for selected inborn errors of metabolism," Journal of the American College of Nutrition, vol. 8, no. 1, pp. 54S-60S, 1989.
[5] A. MacDonald, J. C. Rocha, M. van Rijn, and F. Feillet, "Nutrition in phenylketonuria," Molecular Genetics and Metabolism, vol. 104, pp. S10-S18, 2011.

[6] R. A. Williams, C. D. S. Mamotte, and J. R. Burnett, "Phenylketonuria: an inborn error of phenylalanine metabolism," Clinical Biochemist Reviews, vol. 29, no. 1, pp. 31-41, 2008.

[7] A. MacDonald, H. Gokmen-Ozel, M. van Rijn, and P. Burgard, "The reality of dietary compliance in the management of phenylketonuria," Journal of Inherited Metabolic Disease, vol. 33, no. 6, pp. 665-670, 2010.

[8] A. Aguiar, K. Ahring, M. F. Almeida et al., "Practices in prescribing protein substitutes for PKU in Europe: no uniformity of approach," Molecular Genetics and Metabolism, vol. 115, no. 1, pp. 17-22, 2015.

[9] F. J. van Spronsen and P. Burgard, "The truth of treating patients with phenylketonuria after childhood: the need for a new guideline," Journal of Inherited Metabolic Disease, vol. 31, no. 6, pp. 673-679, 2008.

[10] B. Chabance, P. Marteau, J. C. Rambaud et al., "Casein peptide release and passage to the blood in humans during digestion of milk or yogurt," Biochimie, vol. 80, no. 2, pp. 155-165, 1998.

[11] D. M. Ney, S. T. Gleason, S. C. van Calcar et al., "Nutritional management of PKU with glycomacropeptide from cheese whey," Journal of Inherited Metabolic Disease, vol. 32, no. 1, pp. 32-39, 2009.

[12] P. Solverson, S. G. Murali, A. S. Brinkman et al., "Glycomacropeptide, a low-phenylalanine protein isolated from cheese whey, supports growth and attenuates metabolic stress in the murine model of phenylketonuria," American Journal of Physiology: Endocrinology and Metabolism, vol. 302, no. 7, pp. E885-E895, 2012.

[13] S. C. van Calcar, E. L. MacLeod, S. T. Gleason et al., "Improved nutritional management of phenylketonuria by using a diet containing glycomacropeptide compared with amino acids," American Journal of Clinical Nutrition, vol. 89, no. 4, pp. 1068-1077, 2009.

[14] S. C. van Calcar and D. M. Ney, "Food products made with glycomacropeptide, a low-phenylalanine whey protein, provide a new alternative to amino acid-based medical foods for nutrition management of phenylketonuria," Journal of the Academy of Nutrition and Dietetics, vol. 112, no. 8, pp. 12011210, 2012.

[15] C. E. Laclair, D. M. Ney, E. L. MacLeod, and M. R. Etzel, "Purification and use of glycomacropeptide for nutritional management of phenylketonuria," Journal of Food Science, vol. 74, no. 4, pp. E199-E206, 2009.

[16] R. Matalon, K. Michals-Matalon, G. Bhatia et al., "Double blind placebo control trial of large neutral amino acids in treatment of PKU: effect on blood phenylalanine," Journal of Inherited Metabolic Disease, vol. 30, no. 2, pp. 153-158, 2007.

[17] R. Matalon, K. Michals-Matalon, G. Bhatia et al., "Large neutral amino acids in the treatment of phenylketonuria (PKU)," Journal of Inherited Metabolic Disease, vol. 29, no. 6, pp. 732-738, 2006.

[18] P. Guldberg and F. Guttler, "Mutations in the phenylalanine hydroxylase gene: methods for their characterization," Acta Paediatrica, vol. 83, no. 407, pp. 49-56, 1994.

[19] F. Güttler and H. Lou, "Phenylketonuria and hyperphenylalaninemia," in Inborn Metabolic Diseases: Diagnosis and Treatment, J. Fernandes, J.-M. Saudubray, and K. Tada, Eds., pp. 161-174, Springer, Berlin, Heidelberg, Germany, 1990.

[20] F. Guttler, "Hyperphenylalaninemia: diagnosis and classification of the various types of phenylalanine hydroxylase 
deficiency in childhood," Acta paediatrica Scandinavica. Supplement, vol. 280, pp. 1-80, 1980.

[21] G. N. Thompson and D. Halliday, "Significant phenylalanine hydroxylation in vivo in patients with classical phenylketonuria," Journal of Clinical Investigation, vol. 86, no. 1, pp. 317-322, 1990.

[22] G. N. Thompson, P. J. Pacy, R. W. Watts, and D. Halliday, "Protein metabolism in phenylketonuria and Lesch-Nyhan syndrome," Pediatric Research, vol. 28, no. 3, pp. 240-246, 1990.

[23] J. F. Rehfeld, "Accurate measurement of cholecystokinin in plasma," Clinical Chemistry, vol. 44, no. 5, pp. 991-1001, 1998.

[24] ABX H., Diagnostic test for glucose, 2008.

[25] Diagnostic Test for Blood Urea Nitrogen (BUN), 2014, http:// www.horiba.com.

[26] M. A. Nauck, N. Kleine, C. Ørskov, J. J. Holst, B. Willms, and W. Creutzfeldt, "Normalization of fasting hyperglycaemia by exogenous glucagon-like peptide 1 (7-36 amide) in type 2 (non-insulin-dependent) diabetic patients," Diabetologia, vol. 36, no. 8, pp. 741-744, 1993.

[27] E. Näslund, J. Bogefors, S. Skogar et al., "GLP-1 slows solid gastric emptying and inhibits insulin, glucagon, and PYY release in humans," American Journal of Physiology: Regulatory, Integrative and Comparative Physiology, vol. 277, no. 3, pp. R910-R916, 1999.

[28] S. Andreasen, Ghrelin Elisa Kit, 2015.

[29] Special Assays: Monitoring of Amino Acid on Request, 2016, http://www.zfs-reutlingen.de.

[30] O. K. Zaki, L. El-Wakeel, Y. Ebeid et al., "The use of glycomacropeptide in dietary management of phenylketonuria," Journal of Nutrition and Metabolism, vol. 2016, 5 pages, 2016.

[31] A. Pinto, M. F. Almeida, P. C. Ramos et al., "Nutritional status in patients with phenylketonuria using glycomacropeptide as their major protein source," European Journal of Clinical Nutrition, vol. 71, no. 10, pp. 1230-1234, 2017.

[32] M. Luciana, J. Sullivan, and C. A. Nelson, "Associations between phenylalanine-to-tyrosine ratios and performance on tests of neuropsychological function in adolescents treated early and continuously for phenylketonuria," Child Development, vol. 72, no. 6, pp. 1637-1652, 2001.

[33] Neelima, R. Sharma, Y. S. Rajput, and B. Mann, "Chemical and functional properties of glycomacropeptide (GMP) and its role in the detection of cheese whey adulteration in milk: a review," Dairy Science and Technology, vol. 93, no. 1, pp. 21-43, 2013.

[34] J. Pietz, A. Rupp, F. Ebinger et al., "Cerebral energy metabolism in phenylketonuria: findings by quantitative in vivo 31P MR spectroscopy," Pediatric Research, vol. 53, no. 4, pp. 654-662, 2003.

[35] J. Pietz, R. Kreis, A. Rupp et al., "Large neutral amino acids block phenylalanine transport into brain tissue in patients with phenylketonuria," Journal of Clinical Investigation, vol. 103, no. 8, pp. 1169-1178, 1999.

[36] V. Leuzzi, M. Tosetti, D. Montanaro et al., "The pathogenesis of the white matter abnormalities in phenylketonuria. A multimodal 3.0 tesla MRI and magnetic resonance spectroscopy (1H MRS) study," Journal of Inherited Metabolic Disease, vol. 30, no. 2, pp. 209-216, 2007.

[37] F. J. van Spronsen, M. J. de Groot, M. Hoeksma, D. J. Reijngoud, and M. van Rijn, "Large neutral amino acids in the treatment of PKU: from theory to practice," Journal of Inherited Metabolic Disease, vol. 33, no. 6, pp. 671-676, 2010.

[38] G. M. Knudsen, S. Hasselbalch, P. B. Toft, E. Christensen, O. B. Paulson, and H. Lou, "Blood-brain barrier transport of amino acids in healthy controls and in patients with phenylketonuria," Journal of Inherited Metabolic Disease, vol. 18, no. 6, pp. 653-664, 1995.

[39] S. Schindeler, S. Ghosh-Jerath, S. Thompson et al., "The effects of large neutral amino acid supplements in PKU: an MRS and neuropsychological study," Molecular Genetics and Metabolism, vol. 91, no. 1, pp. 48-54, 2007.

[40] S. Yano, K. Moseley, and C. Azen, "Large neutral amino acid supplementation increases melatonin synthesis in phenylketonuria: a new biomarker," Journal of Pediatrics, vol. 162, no. 5, pp. 999-1003, 2013.

[41] M. Bik-Multanowski and J. J. Pietrzyk, "LAT1 gene variants-potential factors influencing the clinical course of phenylketonuria," Journal of Inherited Metabolic Disease, vol. 29, no. 5, p. 684, 2006.

[42] R. Koch, "Large neutral amino acid therapy and phenylketonuria: a promising approach to treatment," Molecular Genetics and Metabolism, vol. 79, no. 2, pp. 110-113, 2003.

[43] M. K. Jordan, R. L. Brunner, M. M. Hunt, and H. K. Berry, "Preliminary support for the oral administration of valine, isoleucine and leucine for phenylketonuria," Developmental Medicine and Child Neurology, vol. 27, no. 1, pp. 33-39, 1985.

[44] E. L. MacLeod, M. K. Clayton, S. C. van Calcar, and D. M. Ney, "Breakfast with glycomacropeptide compared with amino acids suppresses plasma ghrelin levels in individuals with phenylketonuria," Molecular Genetics and Metabolism, vol. 100, no. 4, pp. 303-308, 2010.

[45] K. H. Schulpis, I. Papassotiriou, M. Vounatsou, G. A. Karikas, S. Tsakiris, and G. P. Chrousos, "Morning preprandial plasma ghrelin and catecholamine concentrations in patients with phenylketonuria and normal controls: evidence for catecholamine-mediated ghrelin regulation," Journal of Clinical Endocrinology and Metabolism, vol. 89, no. 8, pp. 3983-3987, 2004.

[46] D. M. Ney, B. M. Stroup, M. K. Clayton et al., "Glycomacropeptide for nutritional management of phenylketonuria: a randomized, controlled, crossover trial," American Journal of Clinical Nutrition, vol. 104, no. 2, pp. 334-345, 2016. 


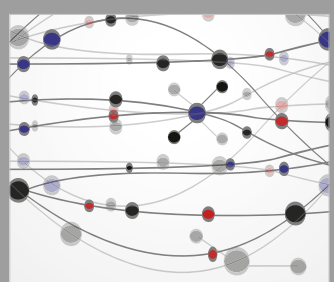

The Scientific World Journal
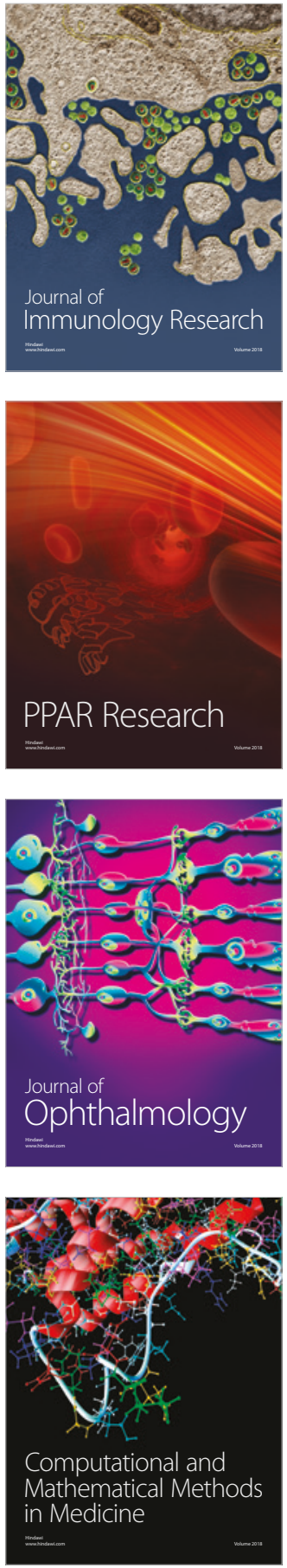

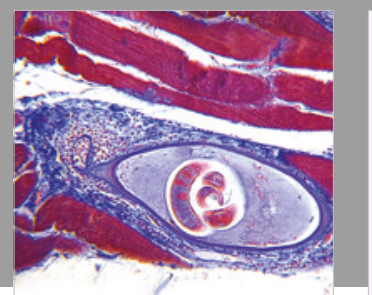

Gastroenterology Research and Practice

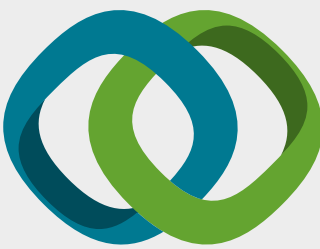

\section{Hindawi}

Submit your manuscripts at

www.hindawi.com
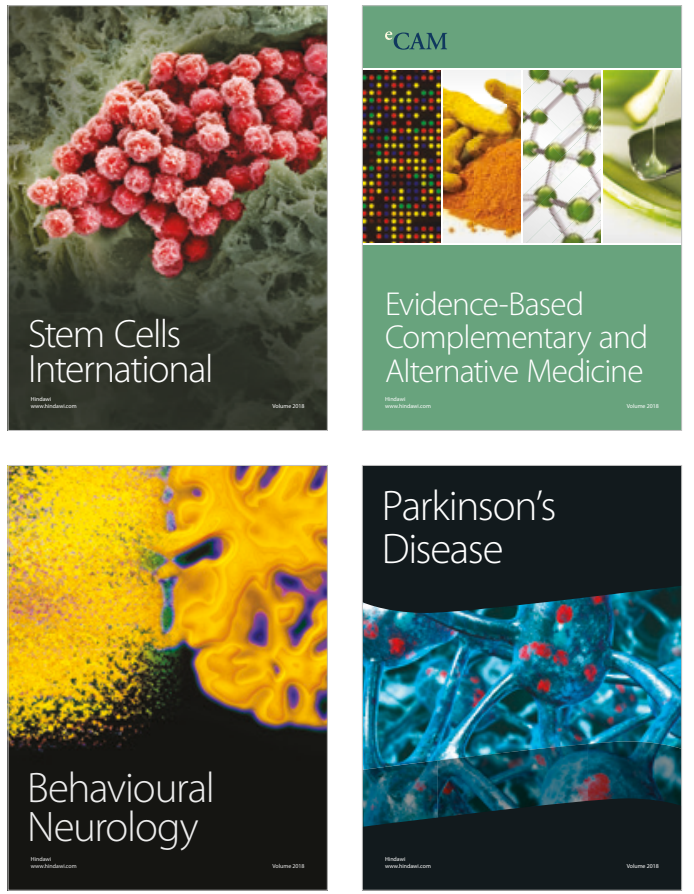

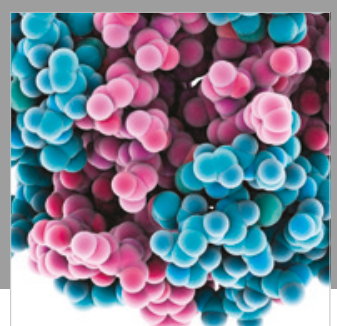

ournal of

Diabetes Research

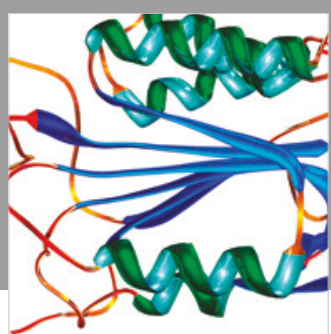

Disease Markers
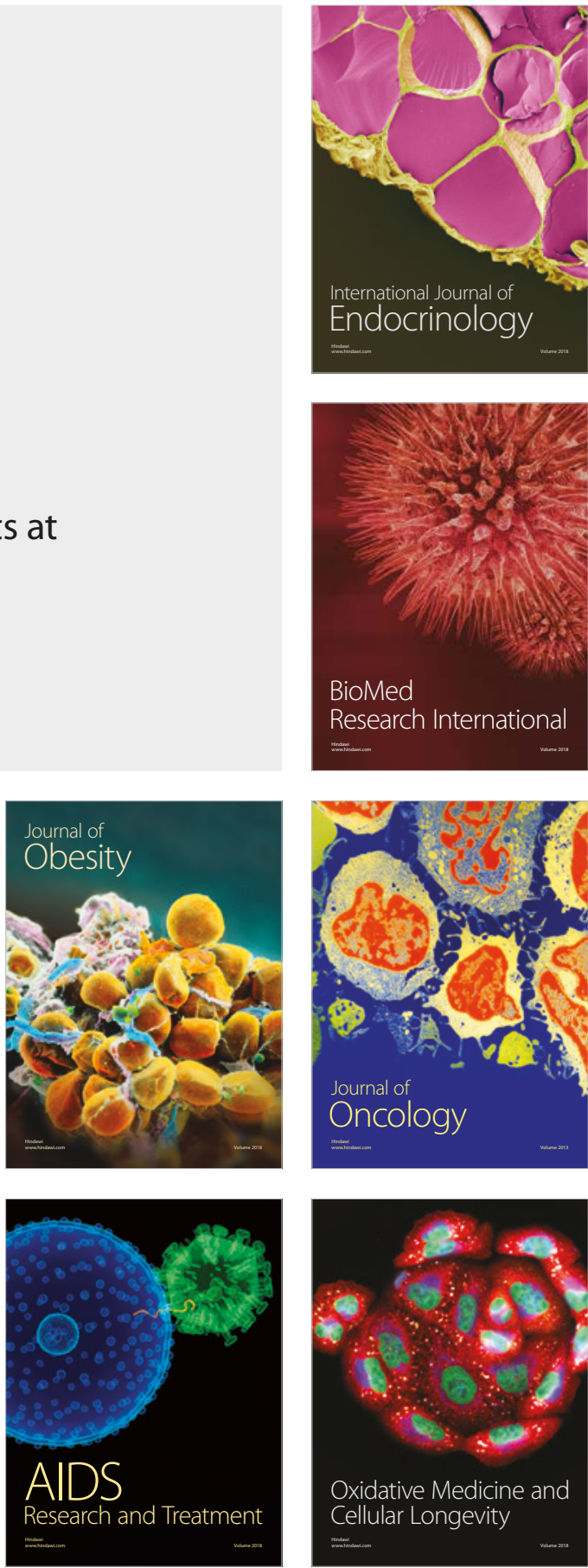